\section{Cosmic rays and interstellar tunnels}

MEASUREMENTs have been made ${ }^{1}$ of the isotopic abundances of $\mathrm{Be}$ in cosmic rays, which have led to the conclusion that the typical cosmic ray lifetime in the galaxy is $\sim 2 \times 10^{7} \mathrm{yr}$. Furthermore, measurements ${ }^{2}$ of the cosmic ray electron energy spectrum in the range $6-100 \mathrm{GeV}$, have shown a bend in the spectrum which can be attributed to inverse Compton and synchrotron losses. The position of the bend is indicative of the cosmic ray electron lifetime, and an age of $1.2 \times 10^{7} \mathrm{yr}$ is derived.

These two values are significantly longer than the widely quoted value of $\sim 3 \times 10^{6} \mathrm{yr}$. This latter value is consistent with the average column density of material traversed by cosmic rays, $\sim 5 \mathrm{~g} \mathrm{~cm}^{-2}$ (as determined by measurements of products of spallation reactions), and the usually assumed average density of matter in the galactic disk $\left(\sim 1 \mathrm{H}\right.$ atom $\left.\mathrm{cm}^{-3}\right)$.

Measurements of the interstellar density indeed show ${ }^{3}$ $\rho_{\text {is }} \sim 1$. The newly determined cosmic ray lifetime, however, implies that the average density through which the particles have travelled is $\sim 0.2$ atom $\mathrm{cm}^{-3}$. This value is an upper limit to the average density encountered by cosmic rays in the galaxy at large, since there is evidence indicating that cosmic rays traverse a large fraction of the above mentioned column density at the particle sources. ${ }^{4}$. It is therefore apparent that cosmic rays must spend $\sim 80 \%$ of their lifetimes in regions of the galaxy where the density is significantly less than the average value found in the disk.

For this reason, the existence of a cosmic ray "halo" has been postulated ${ }^{2,5}$. This halo is a low-matter density region of cosmic ray confinement which surrounds the galactic disk.

Webster ${ }^{6}$, however, analysed data concerning the radio halo of the galaxy, and found that the idea of static confinement in the halo is inconsistent with the observations. Dynamic confinement such as that proposed by Jokipii ${ }^{5}$ may explain the low mean density traversed by cosmic rays.

An alternative explanation is proposed here. The low mean density traversed may be indicative of the presence of hot, low density tunnels in the interstellar medium such as those proposed by Cox and Smith? . Cosmic rays produced in supernovae or in supernova remnants are preferentially injected into these tunnels. The tunnels act as cosmic ray traps in which the density is low $\left(\sim 10^{-3}\right.$ atom $\left.\mathrm{cm}^{-3}\right)$, thus reducing the mean density encountered over the particle lifetime.

Many of the theories of the origin of cosmic rays involve particle acceleration by supernovae explosions, by the resultant pulsar, or in the supernova remnants. Analysis of cosmic ray abundances ${ }^{8}$ supports the view that cosmic rays are accelerated, at least partially, in supernova remnants (compare with ref. 9 and $J$. R. Jokipii, personal communication). Indeed, $\gamma$-ray observations of the Vela supernova remnant ${ }^{10}$ show that it is likely that supernovae or supernova remnants both produce and confine enough cosmic rays to explain the observed galactic cosmic ray density.

Cox and Smith ${ }^{7}$ estimate that a fraction, $f$, of the volume of the interstellar medium is occupied by hot, tenuous tunnels caused by linked chains of supernova remnants, where $0.1 \lesssim f \lesssim 0.5$. Since typical remnants expand to a radius of $\sim 40$ pc before they start to disappear, they have a probability, $P$, of intersection with an existing tunnel, such that $0.6 \lesssim P \lesssim 1$. When the shock front of a new remnant encounters an existing tunnel, the shock is preferentially driven along the tunnel. Thus the remnant material which contains the cosmic rays produced by the supernova (Higdon and Lingenfelter's ${ }^{10}$ data indicate that cosmic rays are confined up to a remnant radius of $\lesssim 30 \mathrm{pc}$ ) is injected into the tunnel system.

As was pointed out previously?, the tunnel systems are excellent cosmic ray traps, since they are regions of low magnetic field. The tunnels are intertwined about the large scale flux tubes of the galactic magnetic field. Particles injected into the system are therefore internally reflected by the high magnetic fields in the tunnel walls. The particles can escape only by slowly diffusing on to the galactic field lines.

The most likely mechanism of particle escape from the tunnel is resonant pitch-angle scattering with turbulent fluctuations in the interstellar field. The typical $(\sim 3 \mathrm{GeV})$ cosmic ray particle has a gyro radius $\sim 10^{12}-10^{13} \mathrm{~cm}$ in the galactic field ( $\sim 3 \times 10^{-6}$ gauss). Unfortunately, we have no observations of the scale size of the turbulence in this regime, and this makes it difficult to estimate the time scale of escape. It is, however, interesting to note that this mechanism will, if operative, give an energy dependence to the cosmic ray spallation products. This is because particles with different gyro radii (that is, different energies) will scatter most effectively off correspondingly different sized fluctuations, and the probability of encountering a given size fluctuation is generally a function of the size of that particular turbulent element. This effect produces a variation in the fraction of the lifetime of the particles which is spent in the high density regions of the disk, and thus a variation in the spallation products. Energy dependence of spallation products is indeed observed ${ }^{11}$, although an alternative explanation has been suggested ${ }^{9}$.

JOHN S. SCOTT

Kitt Peak National Observatory,

Tucson, Arizona 85726

Received August 20; accepted September 11, 1975

Meegan, C. A., and Earl, J. A. (in the press). Jenkins, E. B., and Savage, B. D. N., Astrophys. J. Lett., 184, L47-52 (1973).

Jokipi, $\mathbf{R}$ (in the press).

Webster, A. Mon. Not. R. astr. Soc., 171, 243-257 (1975)

Webster, A., Mon. Not. R. astr. Soc., 171, 243-257 (199, L105-108 (1974)

C. B., and Schramm, D. N., Astrophys. J. (in the

press).
Scott. J.S., and Chevalier, R. A., Astrophys. J. Lett., 197, L5-8 (1975)

10 Higdon, J. C., and Lingenfelter, R. E., Astrophys. J. Lett., 198, L17-20 (1975).

11 Cesarsky, C. J., and Audoze, J., in Proc. 13th Int. Cosmic Ray Conf., 1 (Denver, 1973).

\section{Measurements of the far-field resonance cone for whistler mode waves in a magnetoplasma}

USING a large volume laboratory magnetoplasma the wave fields and resonance cone of the right hand polarised (whistler mode) wave have been mapped at distances of up to 10 whistler wavelengths from a transmitting antenna. The results are in good agreement with theory and suggest that energy is convected away from the antenna along the surface of the resonance cone. Considerable attention has been paid to the generation of waves by a point source in a magnetoplasma, and theoretical analyses ${ }^{1-5}$ have shown that for certain combinations of the antenna operating frequency $\omega$, the electron gyrofrequency $\omega_{c}$, and the plasma frequency $\omega_{\mathrm{p}}$, the fields become singular along a cone whose axis is parallel to $B_{0}$, the steady magnetic field. By examining the refractive index surfaces it can be seen that the group velocity of the wave is zero when the ray vector is parallel to the resonance cone of half angle $\theta$ (when the wave normal is at an angle $\pi / 2-\theta$ to $B_{0}$ ). We restrict ourselves here to a discussion of the radiation pattern for frequencies $\omega<\omega_{r} \ll \omega_{p}$. Waves launched by the antenna then propagate in the right hand polarised whistler mode and the angle of the resonance cone is approximately given by $\theta=\sin ^{-1}\left(\omega / \omega_{\mathrm{c}}\right)$.

This phenomenon is of particular interest because it defines a narrow region of space in a magnetoplasma where the group velocity is very small. The question then arises as to whether energy is, in fact, transferred along the resonance cone or is merely a near-field phenomenon. If 